\title{
Effect of long term simvastatin administration as an adjunct to ursodeoxycholic acid: evidence for a synergistic effect on biliary bile acid composition but not on serum lipids in humans
}

\author{
F Lanzarotto, B Panarotto, R Sorbara, M Panteghini, F Pagani, S Sosta, A Lanzini
}

\begin{abstract}
Background-Stimulated bile acid synthesis preferentially utilises newly synthesised cholesterol, raising the posssiblity that combination of simvastatin (an inhibitor of cholesterol synthesis) with ursodeoxycholic acid (UDCA; a stimulator of bile acid synthesis) may result in reduced bile acid synthesis and greater enrichment of the pool with UDCA than that achieved with UDCA treatment alone.

Aims-To investigate the effect of simvastatin and UDCA given alone and in combination on serum and biliary lipid and biliary bile acid composition.

Methods-Eighteen patients with primary non-familial hypercholesterolaemia were studied during treatment with simvastatin $20 \mathrm{mg} / \mathrm{day}$, UDCA $10 \mathrm{mg} / \mathrm{kg} / \mathrm{day}$, and a combination of the two drugs. Each regimen was given in random order for three months following a three month lead in period.
\end{abstract}

Results-Simvastatin significantly reduced serum low density lipoprotein (LDL) cholesterol but biliary cholesterol concentration remained unchanged. Combination of the two drugs had no synergistic effect on serum cholesterol concentration, but significantly increased the proportion of UDCA in the bile acid pool from $35 \%$ during UDCA to $48 \%$ during combination treatment $(p<0.04)$.

Conclusions-Results showed that: (1) simvastatin reduces serum LDL cholesterol but has no effect on biliary cholesterol concentration, supporting the concept that newly synthesised cholesterol is not the preferential source for biliary cholesterol; and (2) combination of simvastatin with UDCA has the predicted effect of enhancing the proportion of UDCA in the pool. This effect may be of benefit in the treatment of cholestatic liver diseases.

(Gut 1999;44:552-556)

Keywords: simvastatin; HMG-CoA reductase inhibitors; ursodeoxycholic acid; bile; lipids; hypercholesterolaemia; simvastatin

Correspondence to: Dr A Lanzini, 1 Medicina, Spedali Civili, Piazzale Spedali 1, 25100 Brescia, Italy.

Accepted for publication 21 October 1998

It is well documented that administration of statins reduces serum low density lipoprotein (LDL) cholesterol by a mechanism involving inhibition of 3-hydroxy-3-methylglutaryl CoA reductase (HMG-CoA reductase) activity, the rate limiting step in hepatic cholesterol synthesis. ${ }^{1}$ In addition to their effect on serum lipids, statins have also been reported to influence biliary lipid composition, but the evidence for this phenomenon is conflicting. ${ }^{2-13}$ Thus acute administration of simvastatin to cholecystectomised normolipaemic patients has been reported by Loria and colleagues ${ }^{2}$ to reduce hepatic bile acid secretion with no effect on cholesterol secretion. An opposite effect has been reported by Mazzella and colleagues ${ }^{3}$ during short term administration of simvastatin to hypercholesterolaemic patients. Furthermore, the cholesterol saturation index (SI) of gall bladder bile has been reported to be reduced by some $e^{3-6}$ but not all ${ }^{7-11}$ authors during short term administration of simvastatin, pravastatin, or lovastatin. Differences in patient selection, length of treatment, ${ }^{13}$ or pretreatment cholesterol $\mathrm{SI}^{9}{ }^{12}$ may explain these discrepancies, and the effect of statins on biliary lipids is still not certain.

In contrast to the statins, it is well documented that administration of ursodeoxycholic acid (UDCA) reduces biliary cholesterol secretion and cholesterol SI of gall bladder bile. ${ }^{14}$ UDCA is known to cause intestinal cholesterol malabsorption, ${ }^{15}{ }^{16}$ and to stimulate bile acid synthesis from cholesterol as it also causes intestinal bile acid malabsorption. ${ }^{17}{ }^{18}$ It has been suggested that these two effects may increase the hepatic need for cholesterol, thus stimulating the hepatic uptake of LDL, ${ }^{19}$ but reduced serum LDL cholesterol concentration has been an inconsistent finding during UDCA treatment. ${ }^{14} 19$

It is conceivable that when combinations of statins and UDCA are given, the combination of inhibition of cholesterol synthesis with increased bile acid synthesis from cholesterol may have a synergistic effect on both serum and biliary lipid composition; evidence for this latter effect has been reported. ${ }^{36}$ As bile acid synthesis preferentially utilises newly synthesised cholesterol under conditions of stimulated bile acid formation, ${ }^{20}{ }^{21}$ we also reasoned that inhibition of hepatic cholesterol synthesis with simvastatin during stimulation of bile acid

Abbreviations used in this paper: HDL, high density lipoprotein; HMG-CoA reductase, 3-hydroxy-3-methylglutaryl CoA reductase; LDL, low density lipoprotein; SI, saturation index; UDCA, ursodeoxycholic acid; VLDL, very low density lipoprotein. 
synthesis with UDCA may result in greater enrichment of the bile acid pool with UDCA. This is because stimulation of bile acid synthesis is regarded as a main factor limiting accumulation of UDCA in the pool during administration of this bile acid. ${ }^{14}$

The aims of our study were therefore to check the effect of simvastatin and UDCA given alone and in combination on serum and biliary lipid composition, and to test the hypothesis that during administration of a combination of these drugs, enrichment of the pool with UDCA is greater than during treatment with UDCA alone. This latter effect would be of interest in clinical practice because the beneficial effect of UDCA in the treatment of cholestatic liver diseases may depend on the degree of enrichment of the pool with this bile acid.

In order to achieve these aims we carried out a double blind, placebo controlled study with a crossover experimental design comparing the effect on biliary and serum lipid composition of long term treatment with simvastatin alone, UDCA alone, and their combination, given for three months each to patients with primary non-familial hypercholesterolaemia. A carefully monitored dietary lead in period of three months preceded randomisation to treatment in order to ensure steady state conditions prior to the start of the study.

\section{Patients and methods}

PATIENTS

A total of 18 patients (11 men, seven women), aged 38-68 years (mean (SD) 54 (2) years), with a body mass index of $22-26 \mathrm{~kg} / \mathrm{m}^{2}$ (mean 23 (1) $\mathrm{kg} / \mathrm{m}^{2}$ ) was studied. All patients were diagnosed as having primary non-familial hypercholesterolaemia without complications. All patients were gallstone-free as judged by abdominal ultrasonography. No patient was receiving drugs known to influence serum lipid concentrations for three months before entering the study. The study protocol was approved by the local ethics committee, and all patients were asked to sign an informed consent form before entering the study.

\section{EXPERIMENTAL DESIGN AND CLINICAL} PROCEDURE

Selected patients were followed up at monthly intervals for three months prior to randomisation (dietary lead in period) and during administration of simvastatin alone $(20 \mathrm{mg}$ daily), UDCA alone (10 mg/kg/day), and a combination of the two. Each regimen was given in random order for three months according to a double blind study design for the patients $(n=8)$ who agreed to give bile samples in addition to blood samples, and according to a single blind study design for the rest of the patients giving blood samples only. All patients were asked to maintain their dietary habits for the whole length of the study. Blood samples were drawn during the whole study at monthly intervals for measurement of serum lipid concentration in all patients, and bile samples were also collected at the end of the lead in period and of each treatment period in the eight patients giving multiple bile samples.
Gall bladder bile samples were collected by nasoduodenal intubation using intravenous caerulein to stimulate gall bladder contraction as previously described. ${ }^{22}$ An aliquot of the darkest bile was immediately diluted with methanol and stored at $-20^{\circ} \mathrm{C}$ together with an aliquot of undiluted bile for subsequent analysis.

\section{LABORATORY METHODS}

Bile acid, cholesterol, and phospholipid concentrations in bile samples were measured enzymatically as previously described. ${ }^{162}$ Biliary bile acid composition was measured by HPLC as described by Ruben et al. ${ }^{24}$ In brief, bile acids were extracted from whole bile samples using silica bonded cartridges (Bond-Elut) and eluted with pure methanol. ${ }^{23}$ Conjugated bile acids were analysed by isocratic high pressure liquid chromatography using a Waters LC Module 1 apparatus equipped with a Waters Bondpack C-18 $10 \mu \mathrm{m}$ column, and methanol:phosphate buffer (1:2) as mobile phase ( $\mathrm{pH} 5.2$, flow rate $1 \mathrm{ml} / \mathrm{min}$, and detection at $200 \mathrm{~nm})$.

Serum cholesterol and triglyceride concentrations were measured using an automated enzymatic technique (Boehringer Mannheim Test Combination Cholesterol and Tryglicerides, respectively). High density lipoprotein (HDL) cholesterol was measured in the supernatant following precipitation of LDL and very low density lipoprotein (VLDL) cholesterol. LDL cholesterol was calculated using the formula of Friedewald and Levy. ${ }^{25}$ Serum apolipoprotein A-I and B concentrations were measured by using the radial immunodiffusion technique. ${ }^{26}$

CALCULATION AND EXPRESSION OF RESULTS Cholesterol SI of gall bladder bile was calculated using Carey's critical tables assuming total lipid concentration at $10 \mathrm{~g} / \mathrm{dl} .{ }^{27}$ The Carey's correction factor for percentage UDCA in bile was also applied. ${ }^{28}$ The hydrophobicity index of biliary bile acids was calculated as described by Heuman. ${ }^{29}$ The serum atherogenic index was calculated as LDL:HDL cholesterol ratio.

Results are reported as mean (SEM). Differences were tested for statistical significance using the Student's paired $t$ test, and a $\mathrm{p}$ value less than 0.05 was accepted as indicating statistical significance. ${ }^{30}$

\section{Results}

All 18 patients admitted to the study completed the study period and regularly attended the clinic for blood sample collections. Three of the eight patients enrolled for the biliary lipid study missed one of the four scheduled bile collections (at baseline, during simvastatin, and during simvastatin + UDCA, in individual patients respectively) due to lack of compliance with repeated nasoduodenal intubations.

EFFECT ON SERUM LIPIDS AND ATHEROGENIC INDEX

Total serum cholesterol and triglyceride concentrations remained stable during the three months dietary lead in period (table 1). The 
Table 1 Effect of simvastin and UDCA on serum lipids and the atherogenic index

\begin{tabular}{|c|c|c|c|c|c|c|c|}
\hline & Enrolment & $\begin{array}{l}\text { Lead in } 1 \\
\text { month }\end{array}$ & $\begin{array}{l}\text { Lead in } 2 \\
\text { months }\end{array}$ & $\begin{array}{l}\text { Lead in } 3 \\
\text { months }\end{array}$ & Simvastatin & $U D C A$ & $\begin{array}{l}\text { Simvastatin + } \\
\text { UDCA }\end{array}$ \\
\hline Cholesterol (mg/dl) & $303(12)$ & $303(13)$ & $310(10)$ & $308(12)$ & $237(16)^{\star}$ & $297(12)$ & $253(14)^{\star}$ \\
\hline LDL:HDL ratio & & & & $3.7(0.3)$ & $2.2(0.3) \ddagger$ & $3.0(0.3)$ & $2.3(0.3) \ddagger$ \\
\hline Triglycerides $(\mathrm{mg} / \mathrm{dl})$ & $219(54)$ & $207(34)$ & $197(24)$ & $201(28)$ & $149(30) \S$ & $216(35)$ & $174(24)$ \\
\hline Apolipoprotein A-I (mg/dl) & & & & $155(7)$ & $157(10)$ & $169(7)$ & $154(11)$ \\
\hline Apolipoprotein B (mg/dl) & & & & $165(15)$ & $125(16) \dagger$ & $151(11)$ & $126(16) \dagger$ \\
\hline
\end{tabular}

Results expressed as mean (SEM).

${ }^{\star} \mathrm{p}<0.0001,+\mathrm{p}<0.002, \neq \mathrm{p}<0.003, \$ \mathrm{p}<0.03$ compared with pretreatment.

mean value for the total serum cholesterol concentration decreased from 308 (12) $\mathrm{mg} / \mathrm{dl}$ at enrolment to $237(16) \mathrm{mg} / \mathrm{dl}$ at three months during simvastatin $(\mathrm{p}<0.0001)$, and remained unchanged at 297 (12) $\mathrm{mg} / \mathrm{dl}$ (NS) during treatment with UDCA. The serum atherogenic index as expressed by the LDL:HDL ratio was reduced during simvastatin, but UDCA given alone or in combination had no effect (table 1 and fig 1).

The mean value for the serum triglyceride concentration decreased during simvastatin in comparison with the pretreatment value, but did not significantly change during UDCA given alone or in combination with simvastatin (table 1). The mean value for the apolipoprotein A-I concentration remained unchanged during each of the three regimens studied. The mean value for apolipoprotein B decreased significantly during simvastatin treatment in comparison with the pretreatment value (125 (16) versus 165 (15) $\mathrm{mg} / \mathrm{dl}$, respectively, $\mathrm{p}<0.002$ ), but UDCA given alone or in combination had no effect (table 1).

EFFECT ON BILIARY LIPID AND CHOLESTEROL SATURATION INDEX

Figure 2 shows values for the cholesterol SI of gall bladder bile in individual subjects. The mean value was $1.20(0.10)$ before treatment and remained virtually unchanged at 1.08 (0.12) during simvastatin (NS). The cholesterol SI was significantly lower during UDCA than before treatment $(0.83(0.08)$ versus 1.20 $(0.10)$, respectively, $\mathrm{p}<0.02)$, and was further decreased during combination treatment $(0.54$ $(0.01) ; \mathrm{p}<0.02$ in comparison with pretreatment, and $\mathrm{p}<0.05$ in comparison with UDCA alone).

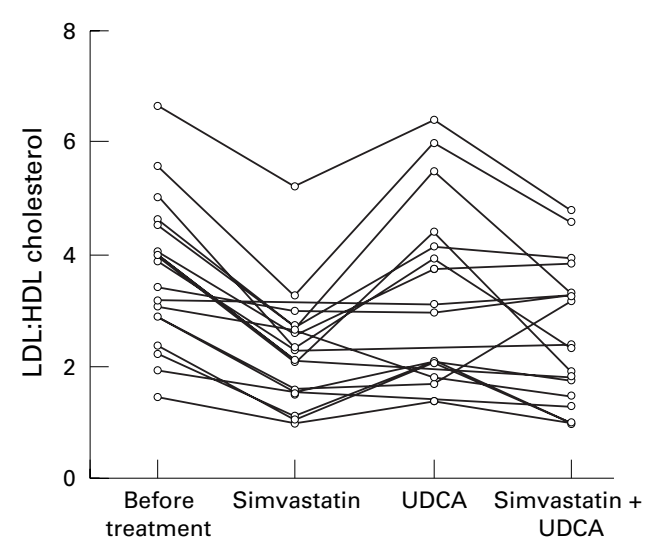

Figure 1 Effect of simvastatin and UDCA given alone or in combination on the atherogenic index (LDL:HDL cholesterol ratio) in individual patients.
EFFECT ON BILIARY BILE ACID AND HYDROPHOBICITY INDEX

Biliary bile acid composition was not affected by simvastatin (table 2). In contrast, the percentage of UDCA in gall bladder bile significantly increased from a mean value of $2.5(0.3) \%$ pretreatment to 35.2 (5.3)\% during UDCA $(p<0.004)$. The enrichment of bile with UDCA further increased to 47.6 (4.9)\% during combination treatment $(p<0.002$ in comparison with pretreatment), and this value was also significantly higher than that observed during UDCA alone $(\mathrm{p}<0.04)$. This effect of UDCA alone and in combination with simvastatin on the percentage of UDCA in bile was mirrored by a significant reduction in both cholic and chenodeoxycholic acid concentrations (table 2).

The mean value for the hydrophobicity index of biliary bile acids was 0.32 (0.02) before treatment; it was not affected by simvastatin $(0.30(0.01), \mathrm{NS})$ but it was significantly reduced during UDCA treatment $(0.08(0.04)$, $\mathrm{p}<0.002$ in comparison with pretreatment). Combination of UDCA with simvastatin further reduced the hydrophobicity index $(-0.01$ $(0.04)$ ), but the difference with UDCA alone did not reach statistical significance.

The cholesterol SI of gall bladder bile was positively related to the hydrophobicity index of biliary bile acids $(y=1.5229 x+0.6388$; $r=0.697, \mathrm{p}<0.001$ ), but there was no relation between the latter and the atherogenic index.

\section{Discussion}

Our study aimed primarily to check whether simvastatin and UDCA have a synergistic effect on serum and biliary lipid concentrations and on bile acid composition by comparing their effect when given in combination with

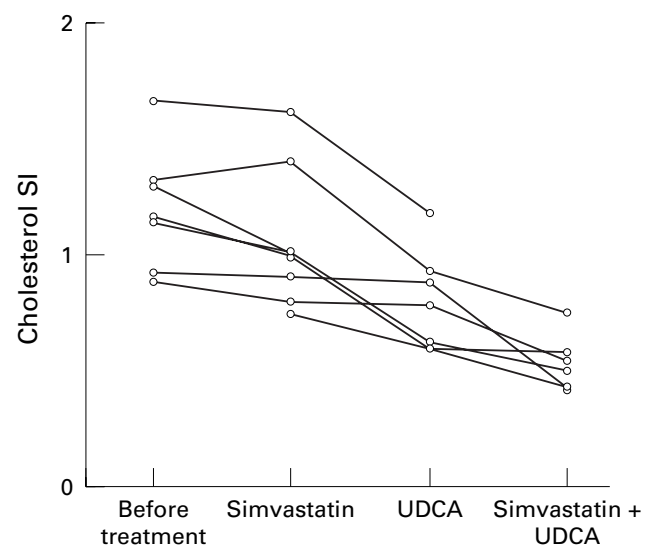

Figure 2 Effect of simvastatin and UDCA given alone or in combination on the cholesterol SI of gall bladder bile in individual patients. 
Table 2 Effect of simvastatin and UDCA on bile acids in gall bladder bile

\begin{tabular}{lllll}
\hline & Pretreatment & Simvastatin & UDCA & Simvastatin + UDCA \\
\hline UCA & $0.4(0.1)$ & $0.4(0.1)$ & $0.5(0.1)$ & $0.7(0.2)$ \\
UDCA & $2.5(0.3)$ & $2.0(0.3)$ & $35.2(5.3) \ddagger$ & $47.6(4.9) \ddagger \subseteq$ \\
CDCA & $34.0(2.2)$ & $33.0(1.5)$ & $21.7(1.8) \dagger$ & $21.7(1.9) \ddagger$ \\
CA & $41.0(3.6)$ & $45.5(2.5)$ & $25.8(3.2) \ddagger$ & $17.4(3.3) \ddagger \star \star$ \\
DCA & $21.2(2.4)$ & $18.0(2.6)$ & $15.3(1.2)$ & $11.4(2.4)^{\star}$ \\
LCA & $1.0(0.2)$ & $1.1(0.3)$ & $1.9(0.2)$ & $1.5(0.6)$ \\
Glyco-conjugated & $70.3(4.2)$ & $72.4(3.2)$ & $76.6(7.9)$ & $84.3(2.6) \dagger$ \\
Tauro-conjugated & $29.7(4.2)$ & $27.3(3.1)$ & $16.2(1.9) \dagger$ & $15.6(2.6)^{\star}$ \\
\hline
\end{tabular}

UCA, ursocholic acid UDCA, ursodeoxycholic acid; CDCA, chenodeoxycholic acid; CA, cholic acid; DCA, deoxycholic acid; LCA, lithocholic acid.

${ }^{\star} \mathrm{p}<0.04,+\mathrm{p}<0.02, \neq \mathrm{p}<0.01$ compared with pretreatment; $\mathbb{\mathrm { p }}<0.04 ;{ }^{\star \star} \mathrm{p}<0.02$ compared with UDCA.

that when given alone. The well known ability of simvastatin to reduce total serum cholesterol and apolipoprotein B concentration was confirmed in our study. This effect was mainly due to a reduction in serum LDL concentrations resulting in a reduced LDL:HDL cholesterol ratio (fig 1), a reduction that has been reported to be associated with an antiatheroma effect. ${ }^{31}$ In contrast with simvastatin, UDCA given alone had no effect on total serum cholesterol or on the LDL:HDL cholesterol ratio. This observation is similar to that obtained in studies involving long term UDCA treatment for gallstone dissolution using the conventional $10-12 \mathrm{mg} / \mathrm{kg} /$ day UDCA dose. ${ }^{14}$ In contrast with these observations, Eusufzai and colleagues ${ }^{19}$ have reported reduced LDL cholesterol in five healthy normolipaemic subjects treated with $30 \mathrm{mg} / \mathrm{kg} /$ day UDCA, and a similar trend for $15 \mathrm{mg} / \mathrm{kg} /$ day UDCA. Taken together these results raise the possibility of a dose response effect of UDCA on serum cholesterol, and suggest that very high dose UDCA may be necessary to stimulate bile acid synthesis to the point that the increased hepatic need of cholesterol is matched by increased uptake of LDL cholesterol.

We also tested the effect of inhibiting HMGCoA reductase with simvastatin on biliary cholesterol concentrations and found no effect (fig 2). This observation is in keeping with the concept that cholesterol for biliary secretion is derived mainly from a preformed pool of serum HDL rather than from de novo hepatic synthesis. ${ }^{32}$ Supporting evidence for this concept has been elegantly provided by recent work of Hillebrant and colleagues ${ }^{33}$ in a study involving measurement of biliary lipid secretion in cholecystectomised patients during removal of serum LDL by apheresis and preservation of HDL. In this study the acute reduction in serum LDL concentration was accompanied by decreased bile acid and phospholipid secretion, and by unchanged cholesterol secretion. Unchanged HDL cholesterol and decreased serum LDL concentrations have also been reported by Loria and colleagues, ${ }^{2}$ to be accompanied by unchanged cholesterol and decreased bile acid secretion in bile during acute simvastatin administration in cholecystectomised patients. Furthermore, increased biliary cholesterol secretion has been observed by Kozarsky and colleagues ${ }^{34}$ using a murine model with an overexpressed HDL receptor enhancing hepatic uptake of this lipoprotein. These results are consistent with our finding that inhibition of hepatic cholesterol synthesis has little effect on biliary cholesterol secretion, and with the concept that unesterified cholesterol carried as HDL is the preferential source of biliary cholesterol.

The lack of effect of simvastatin in reducing biliary cholesterol SI observed in the present and in other studies is indirectly supported by the clinical observation that chronic administration of simvastatin has been reported by van Erpecum and colleagues ${ }^{35}$ to have no effect on dissolution of small radiolucent gallstones. The reason why other authors have reported an effect of simvastatin in reducing biliary cholesterol secretion or concentration ${ }^{3-6}$ is unclear. Differences in experimental conditions may help to explain these discrepancies. Thus, for example, the same group of investigators have reported decreased and unchanged biliary cholesterol secretion during simvastatin administration in hypercholesterolaemic patients ${ }^{3}$ and in obese subjects during weight reduction ${ }^{36}$ respectively.

Combination of simvastatin significantly increased the proportion of UDCA in the pool in comparison with UDCA alone (table 2). Although these results are based on bile analysis in only six patients, the evidence for a synergistic effect of simvastatin and UDCA on the proportion of UDCA in the pool is strengthened by the observation that this effect was present in each patient. The size of this synergistic effect was not very large, but it was large enough to influence the cholesterol SI of gall bladder bile, which was lower during combination therapy than during UDCA alone. This finding of an increased proportion of UDCA in the pool during combination therapy is novel and the mechanism involved is unknown. A possible interpretation is based on the observations that, as mentioned above, chronic UDCA administration is known to stimulate bile acid synthesis, ${ }^{17}{ }^{18}$ an effect regarded as the cause of the limited enrichment of the pool with UDCA. Studies in rats with biliary diversion indicate that stimulated bile acid synthesis preferentially utilises newly synthesised cholesterol. ${ }^{20}$ Results consistent with this concept have been reported by Hillebrandt and colleagues ${ }^{21}$ in cholecystectomised patients with biliary diversion studied before and after inhibition of cholesterol synthesis by pravastatin. These authors reported increased secretion of bile acids and phospholipid but no change in cholesterol secretion during increased cholesterol synthesis at cessation of pravastatin, a finding consistent with a high dependency of bile acid synthesis on de novo cholesterol synthesis under conditions of increased demand for bile acids.

In conclusion, our study indicates that a standard UDCA dose of $10 \mathrm{mg} / \mathrm{kg} /$ day has no synergistic effect with simvastatin in reducing serum LDL concentration. Simvastatin has no effect on biliary lipid composition when given alone, but combination with UDCA results in greater enrichment of the bile acid pool with this bile acid in comparison with UDCA given alone. This latter effect may be important in clinical practice for treatment of cholestatic 
liver diseases, and needs to be confirmed in an adequate number of patients with conditions such as primary biliary cirrhosis where the benefit of UDCA is likely to depend on the extent of enrichment of the pool with UDCA. ${ }^{37}$

1 Grundy SM. HMG-CoA reductase inhibitors for treatment of hypercholesterolemia. N Engl F Med 1988;319:24-32.

2 Loria P, Bertolotti M, Cassinadri MT, et al. Short-term effects of simvastatin on bile acid synthesis and bile lipid secretion in human subjects. Hepatology 1994;19:882-8.

secretion in human subjects. Hepatology 1994;19:882-8. ursodeoxycholic acid and simvastatin plus ursodeoxycholic ursodeoxycholic acid and simvastatin plus ursodeoxycholic acid on biliary lipid secretion and cholic acid kinetics in
nonfamilial hypercholesterolemia. Hepatology 1992;15: nonfamilial

4 Duane WC, Hunninghake DB, Freeman ML, et al. Simvastatin, a competitive inhibitor of HMG-CoA reductase, lowers cholesterol saturation index of gallbladder bile. Hepatology $1988 ; 8: 1147-50$.

5 Mitchell JC, Logan GM, Stone BG, et al. Effects of lovastatin on biliary lipid secretion and bile acid metabolism in humans. F Lipid Res 1991;32:71-8

6 Logan G, Duane WC. Lovastatin added to ursodeoxycholic acid further reduces biliary cholesterol saturation. Gastroenterology 1990;98:15672-6.

7 Smit JWA, van Erpecum KJ, Renooij W, et al. The effects of the 3-hydroxy, 3-methylglutaryl coenzyme A reductase inhibitor pravastatin on bile composition and nucleation of cholesterol crystals in cholesterol gallstone disease. Hepatology 1995;21:1523-9.

8 Smit JWA, van Erpecum KJ, Portincasa P, et al. Effects of simvastatin and cholestyramine on bile lipid composition
and gallbladder motility in patients with hypercholesteroland gallbladder motility in pat

9 Wilson IR, Hurrell MA, Pattinson NR, et al. The effect of simvastatin and bezafibrate on bile composition and gall-bladder emptying in female non-insulin-dependen diabetics. f Gastroenterol Hepatol 1994; 9:447-51.

10 Horiuchi I, Ohya T, Tazuma S, et al. Effects of pravastatin CS-514) on biliary lipid metabolism in patients with hyperlipidemia. Metabolism 1991;40:226-30

11 Reihner E, Rudling M, Stahlberg D, et al. Influence of pravastatin, a specific inhibitor of HMG-CoA reductase, on hepatic metabolism of cholesterol. N Engl f Med 1990; 323 . $224-8$

12 Hoogerbrugge-vd Linden N, de Rooy FWM, Jansen H, et al. Effect of pravastatin on biliary lipid composition and bile acid synthesis in familial hypercholesterolaemia. Gut 1990; acid synthesis

13 Tazuma S, Takizawa I, Kunita T, et al. Effects of long-term treatment with low-dose pravasatatin on biliary lipid and bile acid composition in patients with nonfamilial hyperlibile acid composition in patients with nonfan

14 Bachrach WH, Hofmann AF. Ursodeoxycholic acid in the treatment of cholesterol cholelithiasis. Dig Dis Sci 1982;27: 737-61,833-56.

15 Leiss O, von Bergmann K, Streicher U, et al. Effect of three different dihydroxy bile acids on intestinal cholestero absorption in normal volunteers. Gastroenterology 1984;87: $14-19$

16 Lanzini A, Northfield TC. Effect of ursodeoxycholic acid on biliary lipid coupling and on cholesterol absorption during fasting and eating in subjects with cholesterol gallstones. Gastroenterology 1988;95:408-16.

17 Nilsell K, Angelin B, Leijd B, et al. Comparative effects of ursodeoxycholic acid and chenodeoxycholic acid on bile acid kinetics and biliary lipid secretion in humans. Evidence for a different mode of action on bile acid synthesis. Gastroenterology 1983;85:1248-56.
18 Angelin B, Nilsell K, Einarsson K. Ursodeoxycholic acid treatment in humans: effects on plasma and biliary lipid metabolism with special reference to very low density lipoprotein triglyceride and bile acid kinetics. Eur $\mathcal{F}$ Clin Invest 1986;16:169-77.

19 Eusufzai S, Ericsson S, Cederlund T, et al. Effect of ursodeoxycholic acid treatment on ileal absorption of bile acids in man as determined by the SeHCAT test. Gut 1991;32: 1044-8.

20 Bjorkem I, Lewenhaupt A. Preferential utilisation of newly synthesised cholesterol as substrate for bile acid biosynthesis. F Biol Chem 1979;254:5252-6.

21 Hillebrant CG, Eriksson M, Nyberg B, et al. Changes in biliary lipid output after interruption of pravastatin treatment ary lipid output after interruption of pravasta
in humans. Eur $\mathcal{F}$ Clin Invest 1996;26;1160-5.

22 Lanzini A, Pigozzi G, Facchinetti D, et al. Effect of chronic ursocholic acid administration on bile lipid composition and bile acid pool size in gallstone patients. Scand $\mathcal{F}$ Gastroenterol 1990;25:711-19.

23 Lanzini A, Facchinetti D, Northfield TC. Maintenance of hepatic bile acid secretion rate during overnight fasting by bedtime bile acid administration. Gastroenterology 1988;95: 1029-35.

24 Ruben Ath, van Berge-Henegowen GP. A simple reverse phase high pressure liquid chromatographic determination of conjugated bile acids in serum and bile using a new radial compression separation system. Clin Chim Acta 1982;11:941-50.

25 Friedewald WT, Levy RJ. Estimation of the composition of ow-density lipoprotein cholesterol in plasma, without use of preparative ultracentrifuge. Clin Chem 1972;18:499502 .

26 Panteghini M, Bonora R, Pagani F. Comparison of six commercially available procedures for serum apoliprotein A-I determination. Giornale Italiano Chimica Clinica 1988;13: 251-6.

27 Carey MC. Critical tables for calculating the cholesterol saturation of native bile. 7 Lipid Res 1978;19:945-55.

28 Carey MC, Ko G. The importance of total lipid composition in determining cholesterol solubility in bile and the development of critical tables for calculating percent cholesterol saturation with a correction factor for ursodeoxyesterol saturation with a correction factor for ursodeoxy-
cholate riche bile. In: Paumgartenr G, Stiehl A, Gerok W, eds. Biological effects of bile acids. Lancaster: MTP Press, 1986:299-308.

29 Heuman DM. Quantitative estimation of the hydrophilichydrophobic balance of mixed bile salts solutions. $\mathscr{f}$ Lipid Res 1989;30:719-30

30 Armitage P, Perry G. Statistical methods in medical research. 2nd edn. Oxford: Blackwell Scientific, 1987:93-140.

31 MAAS Investigators. Effect of simvastatin in coronary atheroma: the multicentre anti-atheroma study (MAAS). Lancet 1994:244:633-8.

32 Carey MC. Homing-in on the origin of biliary steroids. Gut 1997;41:721-2.

33 Hillebrant CG, Nyberg B, Einarsson K, et al. The effect of serum low density lipoprotein apheresis on the hepatic serum low density lipoprotein apheresis on the

34 Kozarsky KF, Donahee MH, Rigotti A, et al. Overexpression of the HDL receptor SR-B1 alters serum HDL and bile cholesterol levels. Nature 1997;382:414-17.

35 van Erpecum KJ, Smit JWA, van Berge-Henegowen GP. Long-term treatment with simvastatin does not lead to gallstone dissolution in patients with small cholesterol gallstones [abstract]. Gastroenterology 1995;108:A820.

36 Mazzella G, Cipolla A, Villanova N, et al. Changes in biliary lipid secretion and cholic acid kinetics induced by diet, diet plus simvastatin and diet plus ursodeoxycholic acid in obese subjects. Ital 7 Gastroenterol 1995;27:441-5.

37 Jorgensen RA, Dickson ER, Hofmann AF, et al. Characterisation of patients with a complete response to ursodeoxycholic acid. Gut 1995;36:935-8. 